\title{
PERCEPÇÕES DE PESSOAS QUE VIVEM COM HIVIAIDS SOBRE O CUIDADO OFERECIDO NA ATENÇÃO BÁSICA
}

\section{PERCEPTIONS OF PEOPLE LIVING WITH HIV/AIDS ABOUT THE CARE OFFERED IN PRIMARY CARE}

\section{PERCEPCIONES DE PERSONAS QUE VIVEN CON VIH SOBRE LA ATENCIÓN OFRECIDA EN LA ATENCIÓN BÁSICA}

\author{
Fernanda Esmério Pimentel ${ }^{1}$, Claudiomiro da Silva Alonso ${ }^{2}$, Beatriz Francisco Farah ${ }^{3}$, \\ Girlene Alves da Silva ${ }^{4}$
}

Como citar esse artigo: Pimentel FE, Alonso CS, Farah BF, Silva GA. Percepções de pessoas que vivem com HIV sobre o cuidado oferecido na Atenção Básica. Rev Enferm Atenção Saúde, v. 9, n. 2, 75-87, 2020. DOI: 10.18554/reas.v9i2.3961

\begin{abstract}
RESUMO
Objetivo: conhecer as percepções de pessoas que vivem com HIV sobre o cuidado que lhes é oferecido na Atenção Básica. Método: estudo descritivo-exploratório, de abordagem qualitativa, desenvolvido em um Serviço de Assistência Especializada em HIV/Aids, com 16 participantes. Utilizaram-se entrevistas semiestruturadas, analisadas pelos pressupostos da análise de conteúdo. Resultados: os dados foram organizados em três categorias - Atenção Básica: um cenário subestimado para prestação de cuidados em HIV; Medo de ruptura do sigilo: obstáculo para buscar cuidados na Atenção Básica; Perspectiva controversa de cuidado integral sem a participação da Atenção Básica. Conclusões: verificou-se que os participantes consideram a Atenção Básica como um bom serviço, todavia não buscavam cuidados relacionados ao HIV nesse cenário. O medo de ruptura do sigilo é uma barreira para buscar cuidados na Atenção Básica e possuem uma percepção controversa sobre o cuidado integral, acreditando que um único serviço é capaz de suprir as necessidades em saúde.
\end{abstract}

Descritores: Cuidado; HIV; Atenção Básica.

\begin{abstract}
Objective: to know the perceptions of people living with HIV about the care offered to them in Primary Care. Method: a descriptive-exploratory study, with a qualitative approach, developed in a Specialized Care Service in HIV/AIDS, with 16 participants. Semi-structured interviews were used, analyzed by the assumptions of content analysis. Results: data were organized into three categories - Primary Care: an underestimated scenario for HIV care delivery; Fear of breach of confidentiality: obstacle to seeking care in Primary Care; Controversial perspective of comprehensive care without the participation of Primary Care.

\footnotetext{
${ }^{1}$ Enfermeira. Discente do Curso de Mestrado Acadêmico em Enfermagem da Universidade Federal de Juiz de Fora, Juiz de Fora - MG.

${ }^{2}$ Enfermeiro. Discente do Curso de Mestrado Acadêmico em Enfermagem da Universidade Federal de Minas Gerais. Belo Horizonte - MG.

${ }^{3}$ Enfermeira. Doutora em Saúde Coletiva. Docente do Programa de Pós-Graduação em Enfermagem e Saúde Coletiva da Universidade Federal de Juiz de Fora. Juiz de Fora - MG.

${ }^{4}$ Enfermeira. Doutora em Enfermagem. Docente do Programa de Pós-Graduação em Enfermagem e Saúde Coletiva da Universidade Federal de Juiz de Fora. Juiz de Fora - MG.
} 
Conclusions: it was found that the participants consider Primary Care as a good service; however, they did not seek care related to HIV in this scenario. The fear of breach of confidentiality is a barrier to seeking care in Primary Care and they have a controversial perception about comprehensive care, believing that a single service is capable of meeting health needs.

Descriptors: Care; HIV; Basic Attention.

\section{RESUMEN}

Objetivo: Conocer las percepciones de las personas que viven con el VIH sobre la atención que se les ofrece en Atención Primaria. Método: estudio descriptivo-exploratorio, con enfoque cualitativo, desarrollado en un Servicio de Atención Especializada en VIH / SIDA, con 16 participantes. Se utilizaron entrevistas semiestructuradas, analizadas por los supuestos del análisis de contenido. Resultados: los datos se organizaron en tres categorías: Atención primaria: un escenario subestimado para la prestación de atención del VIH; Miedo a la violación de la confidencialidad: obstáculo para buscar atención en atención primaria; Perspectiva controvertida de la atención integral sin la participación de la Atención Primaria. Conclusiones: se encontró que los participantes consideran la Atención Primaria como un buen servicio, sin embargo, no buscaron atención relacionada con el VIH en este escenario. El miedo a la violación de la confidencialidad es una barrera para buscar atención en Atención Primaria y tienen una percepción controvertida sobre la atención integral, ya que creen que un solo servicio es capaz de satisfacer las necesidades de salud.

Descriptores: Cuidado; VIH; Atención básica.

\section{INTRODUÇÃO}

Conhecer a percepção de usuários sobre os cuidados ofertados pela Rede de Atenção à Saúde (RAS) é relevante para fortalecer as ações desenvolvidas para melhoria da qualidade de vida, especialmente quando se trata de doenças crônicas. ${ }^{1}$

Nesta premissa, o Vírus da Imunodeficiência Humana (HIV) surgiu no Brasil, em 1980 e, desde então, um número significativo de pessoas foram infectadas. Estas se tornaram público-alvo de ações em saúde, uma vez que o último boletim epidemiológico do país revelou 43.941 novos casos de HIV, totalizando 966.058 casos detectados entre 1980 e junho de $2019 .^{2}$
Sobre o cuidado desenvolvido para as Pessoas que vivem com HIV (PVHIV), sabe-se que o Sistema Único de Saúde (SUS) reorganizou os fluxos de atendimento após a doença assumir características crônicas e o modelo tradicional, no qual o cuidado ficava restrito aos Serviços de Assistência Especializados (SAE), indicar falhas, sinalizando a necessidade de articulação entre a os serviços da RAS, com foco no cuidado integral. ${ }^{3,4}$

Posteriormente, o Ministério da Saúde priorizou o compartilhamento de cuidados na RAS, assim, além do SAE, a Atenção Básica $(\mathrm{AB})$ passou a integrar a rede de cuidados às PVHIV. ${ }^{3}$ Destarte, desenvolver ações compartilhadas tornou- 
se fundamental para o avanço do cuidado às PVHIV, garantindo-lhes maior acesso ao sistema de saúde e vínculo. ${ }^{4}$

Nesse sentido, a $\mathrm{AB}$, por meio das ações de promoção da saúde e prevenção de doenças, desempenha papel decisivo no cuidado integral às PVHIV, pois possui proximidade, contato e vínculo com a população. $^{4}$

Ademais, a $\mathrm{AB}$ teve suas potencialidades ampliadas, recebeu investimentos e foi reconhecida pelos gestores em saúde como um cenário valoroso no cuidado de PVHIV. Passou a oferecer testes rápidos, aconselhamento pós-diagnóstico, solicitação de exames complementares e de prosseguimento, estratificação de risco, acompanhamento de assintomáticos estáveis, profilaxia para infecções oportunistas, imunização, início da terapia antirretroviral e detecção precoce de falha na terapia. ${ }^{5}$

Consequentemente, as PVHIV experimentaram um modelo de atenção à saúde que preconiza a articulação na RAS. Com isso, surge o interesse da comunidade científica em desvelar o cuidado desenvolvido para as PVHIV na AB. Nessa ótica, estudo revelou que, em âmbito geral, usuários do SUS avaliam positivamente as ações desenvolvidas pela AB. ${ }^{1}$ Entretanto, possuem uma visão fragmentada do cuidado e do adoecimento, pois acreditam que o cuidado integral é protagonizado por Rev Enferm Atenção Saúde [Online]. Ago/Dez 2020; 9(2):75-87 um único serviço, o que desperta a necessidade de novas pesquisas para melhor compreensão desse fenômeno. ${ }^{6}$

Justifica-se este estudo, pois, na literatura, existem lacunas sobre o cuidado ofertado às PVHIV, visto que estudos recentes foram conduzidos sob o olhar de pessoas que não vivem com $\mathrm{HIV}^{1}$ e de profissionais de saúde ${ }^{3,7}$, não oportunizando às PVHIV exporem suas percepções sobre o cuidado ofertado pela $\mathrm{AB}$, suas potencialidades e desdobramentos na RAS.

Além disso, a Agenda Nacional de Prioridades de Pesquisas em Saúde sugere que desvelar as ações e serviços de saúde destinados às PVHIV e suas percepções sobre o cuidado oferecido é uma prioridade científica, que exige reflexão e análise de temáticas como acesso, vinculação e cuidado compartilhado. ${ }^{8}$

Portanto, o objeto desta investigação foi o cuidado ofertado pela $\mathrm{AB}$. O objetivo foi conhecer as percepções das PVHIV sobre o cuidado que lhes é oferecido na $\mathrm{AB}$.

\section{MÉTODO}

Pesquisa descritiva e exploratória, com abordagem qualitativa, desenvolvida no SAE em HIV/Aids de um município da Zona da Mata Mineira. Esse cenário foi escolhido por acolher grande número de PVHIV, facilitando a proximidade com esses usuários. As questões motivadoras deste estudo foram: PVHIV buscam 
cuidados na $\mathrm{AB}$ ? Como as PVHIV percebem o cuidado desenvolvido na $A B$ ?

Em maio de 2016, realizou-se ambiência para conhecer a rotina do serviço e estabelecer relação com os potenciais participantes. A coleta de dados foi realizada entre junho e outubro de 2016. Foram abordadas 18 pessoas, individualmente, no momento da triagem, que era realizada antes do atendimento no serviço, em sala reservada. Os usuários foram convidados a participar do estudo e espontaneamente revelaram o diagnóstico de HIV. Adiante, foram esclarecidos quanto às motivações, aos riscos e benefícios do estudo.

A amostra deste estudo foi do tipo não probabilística por conveniência, composta por 16 participantes que atenderam aos critérios de elegibilidade. ${ }^{9}$ A reincidência dos dados definiu o número de participantes, tendo sido realizadas mais três entrevistas após tal constatação. ${ }^{10}$ Foram incluídas neste estudo PVHIV, de ambos os sexos, maiores de 18 anos e que residiam em bairros contemplados por Unidade Básica de Saúde (UBS). Foram excluídos dois participantes, um por não residir em bairro com ESF e outro por recusa em participar.

Posteriormente, os dados foram coletados após anuência e assinatura do Termo de Consentimento Livre e Esclarecido, por meio de entrevista semiestruturada, conduzida em ambiente confortável e privativo, com duração de 32 a 57 minutos e com gravação do áudio em mídia digital. A primeira etapa da entrevista foi composta de dados pessoais (idade, sexo e tempo de diagnóstico) e a segunda, pel questões: Quais serviços de saúde você buscou após confirmação do diagnóstico? Você procurou a $\mathrm{AB}$ para realizar acompanhamento? Qual sua percepção sobre o cuidado ofertado na $A B$ ? Quais motivos fazem você buscar cuidados na $A B$ ? Quais os motivos que dificultam a busca de cuidado na $\mathrm{AB}$ ?

Foi assegurado o anonimato dos participantes, codificando-os pelas iniciais de seus nomes. A seguir, as entrevistas foram transcritas e armazenadas em banco de dados do programa Word for Windows. Os participantes receberam feedback das entrevistas e, após concordarem com o conteúdo, estas seguiram o percurso analítico.

Os dados foram analisados pelos pressupostos da análise de conteúdo, constituída pelas etapas: pré-análise, exploração do material e tratamento dos resultados com inferências $\mathrm{e}$ interpretações. ${ }^{11}$ Após a transcrição e análise das entrevistas, agruparam-se as percepções dos participantes em unidades temáticas e em categorias.

A pesquisa foi realizada após aprovação do Comitê de Ética Pesquisa da 
Universidade Federal de Juiz de Fora sob o parecer consubstanciado $\mathrm{n}^{\mathrm{o}}$ 1.5570.0022. Respeitaram-se os princípios éticos dispostos na Resolução $n^{\circ}$ 466/12 do Conselho Nacional de Saúde, que regulamenta pesquisas com seres humanos. $^{12}$

\section{RESULTADOS E DISCUSSÕES}

Participaram deste estudo 16 pessoas, das quais oito eram do sexo feminino e oito do masculino. A faixa etária variou entre 27 e 70 anos, com tempo de diagnóstico entre 7 meses e 20 anos.

A partir das falas, emergiram três categorias - Atenção Básica: um cenário subestimado para prestação de cuidados em HIV; Medo de ruptura do sigilo: obstáculo para buscar cuidados na Atenção Básica; Perspectiva controversa de cuidado integral sem a participação da Atenção Básica.

\section{Atenção Básica: um cenário subestimado para prestação de cuidados em HIV}

Esta categoria foi construída a partir dos fragmentos das entrevistas que abordavam os motivos que levavam as PVHIV a buscarem cuidados na $A B$ e suas percepções sobre o cuidado ofertado. Quanto aos motivos, os participantes relataram que buscavam cuidados na $A B$ quando surgiam necessidades relacionadas a condições agudas, crônicas não transmissíveis e de prevenção, consideradas por eles como mais simples, em comparação com as ações desenvolvidas em outros serviços da RAS, subestimando as potencialidades da $\mathrm{AB}$ :

Vou ao posto por conta de uma gripe ou uma coisinha boba. (R.P.F.)

Vou no posto por coisas simples, como controle da diabetes. (A.M.C.M.)

Vou ao posto para fazer controle da pressão, preventivo, exames, trocar receitas, coisas comuns. (J.M.O.L.)

Os participantes denominaram a $\mathrm{AB}$ como "posto" e, ao serem indagados sobre suas percepções quanto ao cuidado oferecido por ela, reiteraram que esta é um cenário qualificado e que oferece bom atendimento, mas não a buscavam para suprir necessidades relacionadas com HIV:

Eu gosto do atendimento do posto, mas não vou para coisas relacionadas ao HIV. (M.A.F.)

$O$ posto é muito bom, gosto da equipe e do atendimento, mas vou para um exame de rotina ou algum encaminhamento para médico de fora, mas coisas do vírus não. (E.L.)

A AB compreende o nível de saúde mais próximo do cotidiano das pessoas. ${ }^{6}$ Portanto, deveria ser a primeira forma de inserir seus usuários na RAS, fornecendo uma atenção ordenada pela resolubilidade e acolhimento das necessidades em saúde dos usuários. ${ }^{4}$

Verifica-se que às PVHIV acessam a $\mathrm{AB}$, mas que esta não é reconhecida por elas 
como um cenário habilitado ao atendimento das necessidades relacionadas ao HIV. Embora pouco reconhecida frente às suas possibilidades de atuação, a $\mathrm{AB}$, ao desenvolver a perspectiva de integralidade, tem divulgado experiências exitosas quanto à implementação do manejo da infecção pelo HIV. ${ }^{3}$

Apesar de não haver estudo recente sobre a percepção de PVHIV quanto ao cuidado ofertado pela $\mathrm{AB}$, pode-se dizer que, em âmbito geral, os usuários do SUS avaliam positivamente os serviços da $A B$. Estudo transversal realizado com mais de 65 mil usuários relatou que 78,9\% destes consideraram o atendimento da $\mathrm{AB}$ bom ou muito bom. ${ }^{1}$

Ressalta-se que as PVHIV são atendidas na $\mathrm{AB}$ por outros motivos, os quais não estão diretamente relacionados ao HIV. Contudo, é importante que a $A B$ atenda necessidades individuais e coletivas em relação ao HIV, consolidando o princípio da integralidade. ${ }^{4}$ Assim, a busca da $\mathrm{AB}$ exclusivamente para suprir necessidades relacionadas a doenças crônicas não transmissíveis subestima variadas atribuições da $\mathrm{AB}$ no cuidado às PVHIV. Portanto, espera-se que haja o compartilhamento do cuidado, estabelecendo fluxo das informações e permitindo que as equipes da $\mathrm{AB}$ sejam capacitadas, executando suas atribuições para prestar cuidados qualificados e resolutivos estabelecidos pela RAS. ${ }^{13}$

Considera-se que as ações de promoção da saúde e prevenção do HIV estão difundidas e que o diagnóstico de novos casos e o acompanhamento dos usuários diagnosticados estão em progresso. Porém, esses processos são recentes, cercados de polêmica e ainda pouco estudados, embora existam políticas e diretrizes que legitimam as competências da $\mathrm{AB}$ na oferta de cuidados às PVHIV. ${ }^{14}$

O desafio está em superar o paradigma de que as ações desenvolvidas pela $\mathrm{AB}$ são simplistas, o que, neste estudo, foi materializado nas falas dos participantes. Logo, a saúde possui múltiplos determinantes e condicionantes e a melhoria de suas condições passa por diversos fatores, dos quais grande parte são abordados pela AB. ${ }^{4}$

Apesar da $\mathrm{AB}$ não protagonizar o cuidado das PVHIV, os participantes a reconhecem como um serviço qualificado na prestação de cuidados agudos, crônicos e de prevenção, exceto para o cuidado em HIV. Assim, infere-se que a subestima das potencialidades da $\mathrm{AB}$ pode ser superada com a articulação entre a RAS e divulgação das atribuições da AB. Salienta-se que esse fato não está relacionado com a ausência de diretrizes, uma vez que o cuidado compartilhado em HIV/Aids é uma prática 
incentivada e prevista no âmbito normativo da AB.

\section{Medo de ruptura do sigilo: obstáculo para buscar cuidados na $\mathrm{AB}$}

Essa categoria surgiu com a indagação sobre os motivos que dificultavam a busca de cuidados na $A B$. Posteriormente, identificaram-se nas entrevistas subjetividades e sentimentos relacionados ao medo de ter seu diagnóstico revelado pelos profissionais da $A B$, sendo o fator principal que desmotiva a busca por cuidados nesse nível de atenção.

Destaca-se que os participantes não narraram experiências pessoais que materializassem a ruptura de sigilo na $A B$, mas consideraram esse cenário como potencial para quebra do sigilo do diagnóstico.

Tenho medo que eles contem para alguém (profissionais da $A B$ ). Eu não quero que ninguém de lá $(A B)$ saiba. (V.V.P.)

Tem pessoas que não guardariam segredo sobre o que a gente tem, por isso, tenho medo de procurar o posto. (M.A.F.)

O sigilo é compreendido como um aspecto essencial no cuidado em HIV/Aids, principalmente no âmbito da $\mathrm{AB}$ e, quando este não está bem estabelecido entre profissionais e usuários, a formação de vínculo e a busca por cuidados tornam-se prejudicados. $^{3}$ Sabe-se que o sigilo é prerrogativa do compromisso ético do profissional de saúde, sendo indispensável que as PVHIV sejam informadas de que o sigilo e a privacidade são direitos dos usuários. $^{4,13}$

A ruptura do sigilo ocupa lugar de barreira, gerando revés ao acesso dos usuários aos serviços de saúde. Logo, os profissionais de saúde possuem missão ética com a sociedade e devem respeitar os norteadores fundamentais da relação de cuidado, pois o compromisso dos profissionais em garantir uma atenção ética definirá a escolha do usuário pelo serviço que melhor despertar sua confiança. ${ }^{5,15}$

Nessa acepção, estudo transversal, conduzido com 156 participantes com HIV, revelou que a preocupação com o sigilo é uma constante no cenário do cuidado de PVHIV e está relacionada com estigma e discriminação. Além disso, a preocupação com a ruptura do sigilo pode impossibilitar a vinculação e retenção de PVHIV nos diversos serviços de saúde. ${ }^{15}$

Pelo fato da $A B$ ser um serviço próximo ao cotidiano dos usuários, estes acreditam que tal fator contribui para a ruptura do sigilo. ${ }^{4,13}$ Alguns participantes destacaram que a proximidade da $\mathrm{AB}$ com suas realidades era algo que potencializava o medo e a insegurança de ter seu diagnóstico revelado. 
O bairro é muito pequeno, tudo pertinho do posto e fica fácil descobrir o que eu tenho. (E.S.)

Nunca procurei o posto, porque são pessoas conhecidas, bairro todo mundo se conhece. (M.A.F.)

Se, por um lado, obter tratamento perto de casa traz vantagens ao usuário, como evitar percorrer grandes distâncias para cuidar da saúde e receber atendimento integral, por outro, a proximidade pode ser um problema na visão das PVHIV, trazendo riscos potenciais, como a ruptura do sigilo do diagnóstico. ${ }^{4}$

Baseado no princípio de territorialização, a $\mathrm{AB}$ é organizada com alto grau de proximidade da população e suas condições de vida. Assim, a literatura aponta o receio das PVHIV em terem o diagnóstico revelado para a comunidade, a partir do compartilhamento de informações e decisões entre a equipe. Isso torna a territorialidade uma ferramenta de possibilidades de cuidado, mas também fonte potencial de exposição, estigmas e preconceitos. ${ }^{17}$

A formação do vínculo com o usuário é a principal potencialidade da AB. Apesar dos desafios enfrentados, quando ela consegue romper as barreiras do preconceito e do medo sentido pelos pacientes, torna-se instrumento importante para a promoção de saúde do indivíduo, pois, ao oferecer suporte físico e emocional, contribui para a qualidade de vida. ${ }^{18}$
A confiança transmitida pelos profissionais da equipe da $\mathrm{AB}$ às PVHIV contribui para o estabelecimento do vínculo e a adesão ao tratamento. Verifica-se a necessidade de processos educativos para as PVHIV e para equipe da $A B$, reafirmando o binômio (direito/dever) quanto ao sigilo do diagnóstico.

\section{Perspectiva controversa de cuidado integral sem a participação da AB}

Essa categoria foi construída a partir das indagações aos participantes sobre quais serviços de saúde haviam buscado após a confirmação do diagnóstico de HIV e se estes procuraram a $\mathrm{AB}$ para realizar acompanhamento. Os achados revelaram que o SAE foi o serviço escolhido pelos participantes após a confirmação do diagnóstico. Essa situação converge com os fluxos de manejo do cuidado em HIV/Aids.

Assim que eu descobri meu diagnóstico, eu procurei o SAE. (M.A.F.)

Quando descobri que estava com essa doença, vim logo para cá [SAE]. (E.L.)

O conhecimento sobre a busca por cuidados após o diagnóstico de HIV/Aids é essencial para compreender as principais barreiras que permeiam a busca de cuidados na $\mathrm{AB}$. Além disso, é importante que as 
atribuições de cada serviço estejam bem definidas e amplamente divulgadas.

\section{O SAE tem como objetivo} proporcionar atendimento de qualidade às PVHIV, com a premissa de desenvolver o cuidado articulado com outros pontos da RAS, visto que um único serviço não detém todos os recursos necessários para atender à demanda em saúde e contribuir para a legitimação do cuidado integral. ${ }^{14}$

Nesse sentido, tanto o SAE quanto a AB são responsáveis pelo cuidado contínuo, no qual as PVHIV devem passar pelo diagnóstico oportuno, vinculação a um serviço de saúde, retenção por meio do acompanhamento, realização de exames periódicos, adesão ao tratamento e supressão da carga viral para alcançarem qualidade de vida. ${ }^{13}$

Portanto, a busca pelo SAE após o diagnóstico não pode ser considerada uma ação que perturba princípios e objetivos da cascata de cuidado contínuo do HIV, pois esse serviço possui atribuições importantes na RAS, todavia não deve ser entendido como único cenário qualificado para ofertar cuidados às PVHIV.

Nessa acepção, investimentos foram realizados para que a $A B$ se tornasse um serviço capacitado para o cuidado às PVHIV, oferecendo, além de ações de promoção e prevenção, testes para o diagnóstico, acolhimento e acompanhamento. ${ }^{4}$ No município do estudo, a normativa estabelece que a $\mathrm{AB}$ está apta a realizar aconselhamento pósteste, realização de testes rápidos para diagnóstico, além do acompanhamento compartilhado com o SAE. ${ }^{15}$

Ao serem questionados se, após o diagnóstico, buscaram a $\mathrm{AB}$ para realizar acompanhamento, os participantes relataram que não e enfatizaram a satisfação com os cuidados desenvolvidos pelo SAE, caracterizando-o como completo e de qualidade. Os atributos desse serviço geraram uma ilegítima sensação de integralidade no cuidado, dado que frequentavam o $\mathrm{SAE}$, mas procuravam a $\mathrm{AB}$ em busca de cuidados relacionados a condições agudas, crônicas não transmissíveis e ações de prevenção, conforme apontado anteriormente.

Não procurei o posto, porque tudo que eu preciso eu tenho no SAE, tenho nutricionista, dentista, médico, psicólogo, aqui é uma unidade completa. (M.A.F.)

Nunca busquei outros serviços de saúde para o HIV, pois o serviço daqui (SAE) é de qualidade. (V.E.F.)

Depois que descobri o diagnóstico, eu fiquei sendo acompanhada aqui (SAE) e não procurei outro serviço para cuidar do HIV. (T.M.O.)

Não vou no posto por causa desse vírus, porque, aqui no SAE, eles me acolheram e a assistência é muito boa, tenho tudo aqui. (V.V.P.) 
Destaca-se que este estudo não tinha como objetivo conhecer percepções sobre o $\mathrm{SAE}$, mas estas surgiram de forma suplementar, quando os participantes justificavam o motivo pelo qual não buscavam a $\mathrm{AB}$ para acompanhamento. $\mathrm{A}$ satisfação com o SAE é algo presente na literatura, mas a concepção de serviço pleno e sem limitações é controversa. Nessa lógica, estudo transversal avaliou a satisfação de PVHIV com o SAE e apontou alto grau de satisfação com os serviços prestados, porém reconheceu que existem pontos negativos no que concerne a informação, apoio, continuidade, cooperação e organização dos serviços. ${ }^{19}$

Neste estudo, percebe-se que os participantes estão retidos em um único ponto da RAS, o que contribui para centralização do cuidado, limitando a percepção das PVHIV sobre as qualidades do cuidado ofertado pela AB. Entende-se que a rede de cuidados em HIV/Aids está estabelecida e a $\mathrm{AB}$ é componente indispensável 4,5,13. Entretanto, esta apresenta fragilidades e ainda não protagoniza o fluxo de atenção e manejo das PVHIV. Apontam-se como obstáculos a desproporção de serviços oferecidos pela $\mathrm{AB}$ para o número de habitantes, comunicação insuficiente nos serviços de diferentes densidades tecnológicas da RAS e necessidade de preparação dos profissionais para atender às demandas referentes ao HIV. ${ }^{20}$

Nesse cenário, novas metas globais foram propostas para 2020, como o compromisso 90-90-90, das quais o Brasil é signatário. Essas metas significam $90 \%$ das PVHIV diagnosticadas, em tratamento e com carga viral indetectável. Entre as estratégias para alcançar metas destaca-se maior participação da $\mathrm{AB} .{ }^{13}$ Nesse processo de transição, a $\mathrm{AB}$ tem responsabilidade com as PVHIV, oferecendo cuidados que abrangem o teste diagnóstico e o acompanhamento dos usuários assintomáticos e estáveis em primeira linha de tratamento. ${ }^{4,13}$

Assim, a acessibilidade e a utilização da $\mathrm{AB}$ potencializam o diagnóstico precoce e, consequentemente, o manejo adequado da infecção pelo HIV, favorecendo a formação de vínculo entre profissionais e PVHIV, que repercute positivamente na percepção destes usuários. Contudo, o investimento no acesso deve ser intensificado pelas políticas públicas e implementado pelos profissionais, para que a $\mathrm{AB}$ possa se tornar resolutiva. ${ }^{20}$

Infere-se que existe necessidade de fortalecer $\mathrm{a} \mathrm{AB}$ para consolidar o cuidado às PVHIV, por meio de capacitação dos profissionais de saúde, melhor alocação de recursos financeiros e avaliação de estratégias de gestão compartilhada do cuidado em HIV/Aids. 


\section{CONCLUSÃO}

O estudo possibilitou conhecer a percepção das PVHIV sobre o cuidado que lhes é oferecido na $\mathrm{AB}$. Verificou-se que elas a consideraram como um bom serviço, todavia não buscavam cuidados relacionados ao HIV nesse cenário, subestimando suas potencialidades.

Além disso, o medo das PVHIV de ruptura do sigilo é uma barreira para a busca de cuidados na $\mathrm{AB}$ e ainda possuem uma percepção controversa sobre o cuidado integral, acreditando que já tinham suas necessidades atendidas em um único serviço de saúde.

Os resultados sinalizaram importantes reflexões, visto que a $\mathrm{AB}$ foi percebida como um cenário limitado no desenvolvimento de cuidados para as PVHIV, mesmo diante dos avanços nas políticas públicas. Logo, os resultados desta pesquisa preenchem uma lacuna existente e contribuem para que ocorram mudanças no modelo de cuidados ofertado nesse cenário, pautadas na concepção de transformação baseada na percepção de PVHIV. Assim, a AB poderá ser percebida e utilizada, contribuindo significativamente com as metas e indicadores de saúde.

Sugerem-se ações de curto prazo, já preconizadas pelas políticas de saúde para qualificar o cuidado às PVHIV e modificar suas percepções. Tais ações se devem pautar em gestão qualificada de pessoas, processo educativo reflexivo dos preceitos éticos profissionais, competências e atribuições da rede de cuidados, desmitificando a crença de que o cuidado integral pode ser alcançado em um único serviço.

Apresenta-se como limitação deste estudo o fato da coleta de dados ter sido realizada no SAE, o que, em dado momento, fez com que as PVHIV externassem suas percepções sobre o SAE, mesmo não sendo este o objetivo. Além disso, a ausência de pesquisas anteriores que apresentassem a percepção de PVHIV sobre o cuidado oferecido a elas na $\mathrm{AB}$ impediu que os resultados deste estudo fossem comparados e os contrassensos discutidos em profundidade.

\section{REFERÊNCIAS}

1. Abreu DMX, Araújo LHL, Reis CMR, Lima AMLD, Santos AF, Jorge AO, et al. Percepção dos usuários sobre o cuidado prestado por equipes participantes do Programa Nacional de Melhoria do Acesso e da Qualidade da Atenção Básica no Brasil. Rev Epidemiol Serv Saúde [Internet]. 2018 [citado em 18 jun 2020]; 27(3):e2017111. doi:/10.5123/S167949742018000300002

2. Ministério da Saúde (Brasil), Secretaria de Vigilância em Saúde. Boletim Epidemiológico HIV/Aids [Internet]. dez 2019 [citado em 18 jun 2020]; (N Esp):170. Disponível em: http://www.aids.gov.br/ptbr/pub/2019/boletim-epidemiologico-dehivaids-2019

3. Colaço AD, Meirelles BHS, Heidemann ITSB, Villarinho MV. O cuidado à pessoa 
que vive com HIV/Aids na Atenção Primária à Saúde. Texto \& Contexto Enferm. [Internet]. 2019 [citado em 18 jun 2020]; 28:e20170339. doi:/10.1590/1980265x-TCE-2017-0339

4. Ministério da Saúde (Brasil), Secretaria de Vigilância em Saúde. Cuidado integral às pessoas que vivem com HIV pela Atenção Básica: manual para a equipe multiprofissional [Internet]. Brasília, DF: Ministério da Saúde; 2017 [citado em 18 jun 2020]. Disponível em:

http://bvsms.saude.gov.br/bvs/publicacoes/ cuidado_integral_hiv_manual_multiprofiss ional.pdf

5. Ministério da Saúde (Brasil), Secretaria de Vigilância em Saúde. Cinco passos para o Manejo do HIV na atenção básica [Internet]. Brasília, DF: Ministério da Saúde; 2017 [citado em 20 jun 2020]. Disponível em: http://www.aids.gov.br/ptbr/pub/2014/5-passos-para-

implementacao-do-manejo-da-infeccaopelo-hiv-na-atencao-basica

6. Palácio MB, Figueiredo MAC, Souza LB. $\mathrm{O}$ cuidado em HIV/AIDS e a atenção primária em saúde: possibilidades de integração da assistência. Psico [Internet]. 2012 [citado em 20 jun 2020]; 43(3):36067. Disponível em: http://revistaseletronicas.pucrs.br/ojs/index .php/revistapsico/article/view/9816/8237

7. Carvalho VKA, Godoi DF, Perini FB, Vidor AC. Cuidado compartilhado de pessoas vivendo com HIV/AIDS na Atenção Primária: resultados da descentralização em Florianópolis. Rev Bras Med Fam Comunidade [Internet]. 2020 [citado em 20 jun 2020];

15(42):2066. doi:/ 10.5712/rbmfc15(42)2066

8. Ministério da Saúde (Brasil), Secretaria de Ciência, Tecnologia e Insumos

Estratégicos. Agenda de prioridades de Pesquisa do Ministério da Saúde [Internet]. Brasília, DF: Ministério da Saúde; 2018. Disponível em: https://bvsms.saude.gov.br/bvs/publicacoes /agenda_prioridades_pesquisa_ms.pdf
9. Nobre FC, Corrêa DA, Neponuceno LH, Nobre LHN, Sousa AJ, Siqueira Filho V. A amostragem na pesquisa de natureza científica em um campo multiparadigmático: peculiaridades do método qualitativo. Rev Espacios [Internet]. 2017 [citado em 24 jun 2020]; 38(2):1-11. Disponível em: https://www.revistaespacios.com/a17v38n 22/a17v38n21p13.pdf

10. Nascimento LCN, Souza TV, Oliveira ICS, Moraes JRMM, Aguair RCB, Silva LF.

Saturação teórica em pesquisa qualitativa: relato de experiência na entrevista com escolares. Rev Bras Enferm. [Internet]. 2018 [citado em 24 jun 2020]; 71(1):243-8. doi:/ 10.1590/0034-7167-2016-0616

11. Bardin L. Análise de conteúdo. São Paulo: Edições 70; 2016. 141p.

12. Conselho Nacional de Saúde (Brasil). Resolução 466, de 12 de dezembro de 2012. Diretrizes e Normas Regulamentadoras de Pesquisas envolvendo seres humanos [Internet]. Brasília, DF: CONEP; 2012 [citado em 25 jun 2020]. Disponível em: https://bvsms.saude.gov.br/bvs/saudelegis/ cns/2013/res0466_12_12_2012.html

13. Ministério da Saúde (Brasil), Secretaria de Vigilância em Saúde. Protocolo clínico e diretrizes terapêuticas para manejo da infecção pelo HIV em Adulto [Internet]. Brasília, DF: Ministério da Saúde; 2018 [citado em 26 jun 2020]. Disponível em: http://www.aids.gov.br/ptbr/pub/2013/protocolo-clinico-ediretrizes-terapeuticas-para-manejo-dainfeccao-pelo-hiv-em-adultos

14. Medeiros LB, Trigueiro DRSG, Silva DM, Nascimento JA, Monroe AA, Nogueira JA, et al. Integração entre serviços de saúde no cuidado às pessoas vivendo com aids: uma abordagem utilizando árvore de decisão. Ciênc Saúde Colet. [Internet]. 2016 [citado em 25 jun 2020]; 21(2):543-52. doi: https://doi.org/10.1590/141381232015212.06102015

15. Prefeitura Municipal de Juiz de Fora, Secretaria Municipal de Saúde, 
Subsecretaria de Atenção Primária em

Saúde. Catálogo de normas: atenção primária à saúde Juiz de Fora. Juiz de Fora, MG: Secretaria Municipal de Saúde; 2016.

16. Primeira MR, Santos WM, Paula CC,

Padoin SMM. Qualidade de vida, adesão e indicadores clínicos em pessoas vivendo com HIV. Acta Paul Enferm [Internet]. 2020 [citado em 26 jun 2020]; 33:eAPE20190141. http://dx.doi.org/10.37689/actaape/2020ao0141

17. Melo EA, Maksud I, Agostini R. Cuidado, HIV/Aids e atenção primária no Brasil: desafio para a atenção no sistema único de saúde? Rev Panam Salud Publica [Internet]. 2018 [citado em 24 jun 2020]; 42:e151. doi:/10.26633/rpsp.2018.151

18. Garbin CAS, Sandre AS, Rovida TAS, Pacheco KTS, Filho ACP, Garbin AJI. O cuidado para pessoas com HIV/Aids sob a ótica de agentes comunitários de saúde. Trab Educ Saúde [Internet]. 2019 [citado em 26 jun 2020]; 17:e0018508. doi:/10.1590/1981-7746-sol00185

19. Guilherme JA, Yamaguchi MU, Massuda EM. Satisfação dos portadores de HIV/AIDS com o serviço de assistência especializada. REME Rev Min Enferm. [Internet]. 2019 [citado em 25 jun 2020]; 23:e-1213. doi:/10.5935/14152762.20190061

20. Kleinubing RE, Padoin SMM, Langendorf TF, Paula CC. Acesso aos serviços de saúde de mulheres com HIV: pesquisa participante. Atas CIAIQ. [Internet]. 2018 [citado em 27 jun 2020]; 2:34-43.

Disponível em: https://proceedings.ciaiq.org/index.php/ciai q2018/article/view/1762/1715

RECEBIDO: 07/09/2019

APROVADO: 03/09/2020

PUBLICADO: $12 / 2020$ 